\title{
The condition monitoring of diesel engines using acoustic signal analysis
}

\author{
Widi Prasetyo, Mudrik Alaydrus \\ Electrical Engineering Major, Faculty of Engineering, Mercu Buana University, Meruya, Jakarta, Indonesia
}

\begin{tabular}{l}
\hline \hline Article Info \\
\hline Article history: \\
Received May 25, 2018 \\
Revised Sep 28, 2018 \\
Accepted Oct 9, 2018 \\
\hline
\end{tabular}

\section{Keywords:}

Acoustic sound

Correlation coefficient

Envelope extraction

Fast fourier transform

Frequency pattern

\begin{abstract}
Engine have become one of the supportive asset for many activity and work, therefore engine need attention for its condition. The easiest way to do is through the acoustic sound of the engine itself. Most of the time, the engine sounds are checked using a traditional way that may causing a debate regarding its condition. This is due to no supportive scientific basis to know about engine condition using their own acoustic sound. A value from its frequency pattern is needed as scientific basis to determine an engine condition using acoustic sound. Method of envelope extraction by hilbert transform, fast fourier transform, and correlation coefficient are used to find that value. A series of tests have been carried out on the values that have been found and the result are promising for telling engine condition, but unfortunately the values has not been able to identify type of damage that occur on the engine.
\end{abstract}

Copyright (c) 2019 Institute of Advanced Engineering and Science. All rights reserved.

\section{Corresponding Author:}

Widi Prasetyo,

Electrical Engineering Major,

Faculty of Engineering,

Mercu Buana University, Meruya, Jakarta, Indonesia.

Email: widi.prasetyo@outlook.com

\section{INTRODUCTION}

Engine have been used as supportive asset on daily life. It support a vast area, from electrical generator to mining equipment. It is not overreact if engine condition need to be maintained so it can operate properly. Engine maintenance can be devide in to two type of maintenance, they are predetermined preventive maintenance(pdPM) and condition based maintenance(CBM) [1]. Engine maintenance using pdPM type is maintenance that done periodically using pre-set time interval to prevent any major damage of engine component from occur. On the other hand, engine maintenance using CBM is focus on monitoring system of engine condition which regulary transmit a6n information that at some point those information are collected on a parameter that can state the engine condition. CBM type have 3 keys element to determine the maintenance of the monitored engine. Those keys are data collection, data processing, and decision making [1-2].

There are some advantages that can be obtain by doing engine condition monitoring. Those advantages are decreasing maintenance cost of an engine as far as $50 \%-80 \%$, decreasing the amount of equipment damages as far as $50 \%-80 \%$, decreasing overtime costs of human resources as far as $20 \%$ $50 \%$, increasing lifetime of an engine as far as $50 \%-60 \%$, and increasing the total productivity as far as $20 \%-30 \%$ [1], [3].

The fastest and easiest way to determine engine condition is through its acoustic sound. It is can be done because engine acoustic sound can relay an information regarding its condition [4-7]. As for example that using sound as source of information are underwater target recognition [8-9]. Acoustic sound that are produce by engine can be categorize as "healthy" if there are no knocking sound, rattling sound, and hunting sound [9]. Therefore engine condition can be known by using acoustic sound of the engine. 
Most of the time, engine condition monitoring through acoustic sound are done in traditional way, which can lead to a debate regarding the engine condition [2]. It is occur because lack of scientic foundation that support the recognition of engine condition using its own acoustic sound. Beside that, it rely on person's long time experiences in engine maintenance division area and that person's hearing ability. Therefore the result of engine monitoring will be subjective [2].

Through this research engine sound will be visualize as frequency pattern and compare those pattern between healthy engine and damaged engine. Next, those frequency pattern will be used to find a characteristic value that can distinguish healthy engine from damaged engine. With this frequency pattern dan characteristic value condition of engine can be maintained.

Similar research have been conducted several time using various method. Among them wavelet transform [5], [8-11] is more popular then the other. Other method such as linier prediction analysis method [4], blind source separation (BSS) method [3], homomorphic analysis method [9] and Hilbert-Huang Transform (HHT) [12] have also been used to conduct research regarding acoustic sound of the engine condition.

Hongjiang He, Chunxia Wang, and Yazhou conduct a research to detect a component damage using wavelet transform by isolating a sound source of damaged component. The result are successful with conclusion that wavelet transform have more resistance to environment noise [5].

R.M. Vilela, J.C. Metrolho, and J.C. Cardoso conduct research to extract a single signal sound source from a mixed signal sound source using BSS method TDSEP algorithm. Although this research are done in a controlled environment laboratory using a simulation of damaged engine component, the result are promising [3].

Li Jun, Dong Xingwen, Song Yaji, and Song Nuan conduct research to extract an engine sound from an environment noise with homomorphic analysis method continued with cepstrum lines to determine the engine condition. Result of the research said that with more cepstrum lines the accuracy to recognize the engine condition will increase as well [9].

Sadeep Kumar Yadav, Kanishka Tyagi, Brijeshkumar Shah, and Prem Kumar Kalra conduct research ot identify engine condition using envelope detection and FFT. Correlation coefficient are used for making a decision whether the engine in healthy condition or damaged condition. Using those method successfully recognize an engine damage type up to $80 \%$ success rate [2].

Qiang Wang and Xuemin Tian conduct research of soft-sensing algorithm using Hilbert-Huang Transform (HHT) and Wavelet Support Vector Machine (WSVM). The application of HHT-WSVM are used as feature extraction as parts of soft-sensing algorithm. The research result suggested that using HHT-WSVM are more accurate compared to other two methods, KPCA-LSSVM and HHT-SVM. This means that HHTWSVM has a better prediction and generalization [12].

Zhongjie Wang, Jingnan Zhang, and Yangchun Liang conduct a research to test noises and vibrations of a motor. The FFT analysis method is used to acquire the noise signal, recording and spectral analysis. This research conclude that motor noise and vibration from this research can be used to recognize the condition of another motor [7].

Using above research journal [2] as an inspiration, this research are using envelope detection and FFT to get the acoustic sound frequency pattern. The decision making are using comparison between frequency pattern of healthy engine acoustic sound and damaged engine acoustic sound.

\section{RESEARCH METHOD}

As general picture, the research method that are used consist of several step from sampling to decision making. The first step are sampling the acoustic sound of an engine. Then continue to process the sampling using MATLAB software to gain the instantaneous amplitude and then using FFT to get the frequency pattern. The decision making process are based on correlation coefficient on comparison of frequency pattern of healthy engine acoustic sound and damaged engine acoustic sound. Figure 1 shows illustration of above steps.

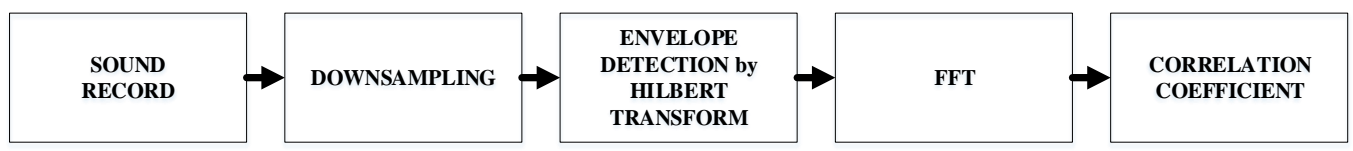

Figure 1. Research step diagram 
Sampling is done by collecting engine acoustic sound that operate at speed $1.500 \mathrm{rpm}$, no load, and using only smartphone microphone. Each sample of engine acoustic sound are recorded with 5 seconds duration and taken at $20 \mathrm{~cm}$ from engine body. Since smartphone microphone mostly have $22.000 \mathrm{~Hz}$ frequency range, the sampling frequency is set at $44.100 \mathrm{~Hz}$ and the recording is saved in WAVE (wav) file. The source of sample that was collected are from various engine maker and model. This is deemed necessary in order to make the engine manufacturer and model not limited just one kind. Table 1 shows the amount of taken sample and Table 2 shows the sources of engine sound samples.

\begin{tabular}{cc} 
Table 1. Sample Quantity \\
\hline Sound Type & Quantity \\
\hline Healthy Sound & 36 \\
Knocking Sound & 23 \\
Hunting Sound & 26 \\
\hline
\end{tabular}

Table 2. Source of Engine Sound Sample

\begin{tabular}{ccccc}
\hline Manufacturer & Model & Running Hour & Application & Capacity \\
\hline CATERPILLAR & D3412 & 55.113 & GENSET & $810 \mathrm{kVA}$ \\
CATERPILLAR & D3512 & 39.662 & COMPRESSOR & $750 \mathrm{kVA}$ \\
CATERPILLAR & D3306 & 42.441 & GENSET & $350 \mathrm{kVA}$ \\
PERKINS & 2006TTAG & 12.773 & GENSET & $450 \mathrm{kVA}$ \\
NISSAN & RD10 & 14.263 & GENSET & $250 \mathrm{kVA}$ \\
VOLVO & TAD 1030 & 15.060 & GENSET & $250 \mathrm{kVA}$ \\
\hline
\end{tabular}

Next, each sample will go through series of process until the frequency pattern is formed. Since the original sample have 5 seconds duration and $44.100 \mathrm{~Hz}$ sampling frequency, it generate approximately 22.000 data point in MATLAB. To ease the computation process and shorten the processing time, only 8.800 data points or equal to 5 times engine rotation will go through next process.

The 8.800 data points are processed with hilbert transform to get the analytic signal, and by doing that the real valued signal is converted into complex signal. The analytic signal or complex signal is necessary to get the signal's envelope detection. The purpose of this envelope detection is to signify any peak that exist in the complex signal. The complex signal consist of 2 parts, real-value signal part and imaginaryvalue signal part. The real part are the signal itself that doesn't go through any process. Another case with the imaginary part, this part is an output of hilbert transformation. The hilbert transformation equation is written as follows [12-15],

$$
H[x(t)]=\tilde{x}(t)=\frac{1}{\pi} \int_{-\infty}^{\infty} \frac{x(\tau)}{t-\tau} d \tau
$$

where $t$ is time, $x(t)$ is time domain signal, and $\tilde{x}(t)$ is the result of hilbert transform as imaginary-value signal part. With real part and imaginary part already known, then the complex signal can be form using following Equation [12-15].

$$
X(t)=x(t)+\tilde{x}(t)=q(t)^{e^{j \varphi(t)}}
$$

The actual form of envelope detection that being use in this research is an absolute value of complex signal itself. The envelope detection or known as instantaneous amplitude have role to signify any amplitude related signal characteristic. In complex signal absolute value equation is written as follows [12-15].

$$
a(t)=|X(t)|=\sqrt{x^{2}(t)+\tilde{x}^{2}(t)}
$$

Next, the signal's envelope detection wil go through FFT process to get into frequency domain. The result of this FFT process always show 2 side of signal, that is positive value and negative value. In this research, only positive value will be used as comparison [16]. The FFT result will be shown on logarithm form to show any detail that exist in the frequency pattern. Figure 2 gives the envelope detection and FFT result. 

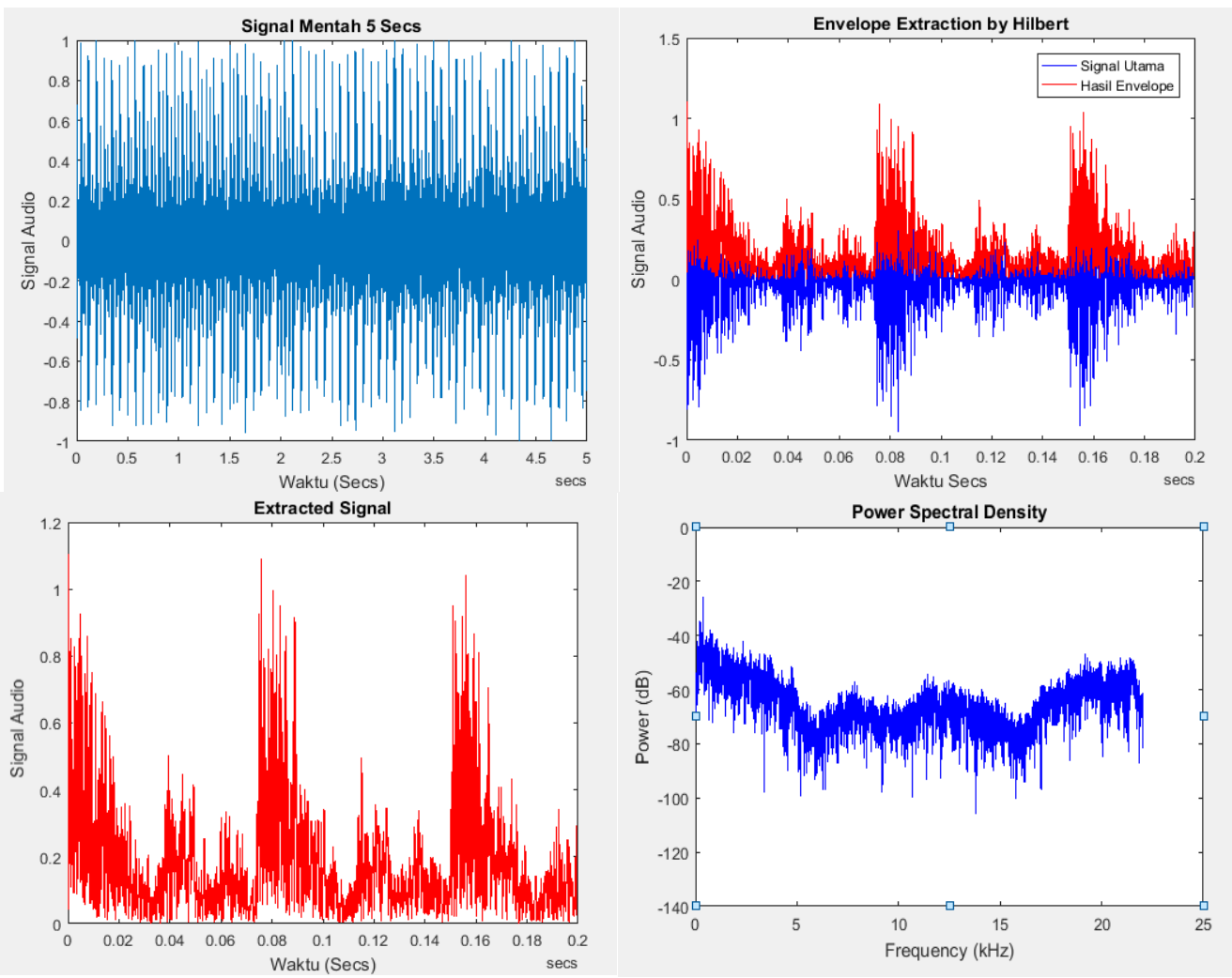

Figure 2. Envelope detection and FFT

The comparison process will be conducted with correlation coefficient method. the aim is to get the value of compared signal in frequency domain and by using that value engine condition can be detected between healthy and damaged. This research is using Pearson correlation coefficient $\rho_{x, y}$ as follows [2],

$$
\rho_{x, y}=\frac{E\left(\left(x-\mu_{x}\right)\left(y-\mu_{y}\right)\right)}{\sigma_{x} \sigma_{y}}
$$

Where $x$ and $y$ is two random variables, $\mu_{x}$ and $\mu_{y}$ is the expected values, $\sigma_{x}$ and $\sigma_{y}$ is the standard deviations, and $E$ is the expected value operator.

A set of healthy engine sound is prepared as a benchmark that each one will be compared with a test engine sound. Then, the output value of each comparison will be averaged and as a result, a single output value wil be generated. This value is used to detecting engine condition. If there are multiple test engine sound, the correlation coefficient will form a matrix that each column have those average value of frequency pattern comparison [17]. Even if the amount of healthy engine sound sample and test engine sound increase, the bacis method of correlation coefficient in this research are still the same. Figure 3 illustrates the correlation coefficient method and formed matrix.
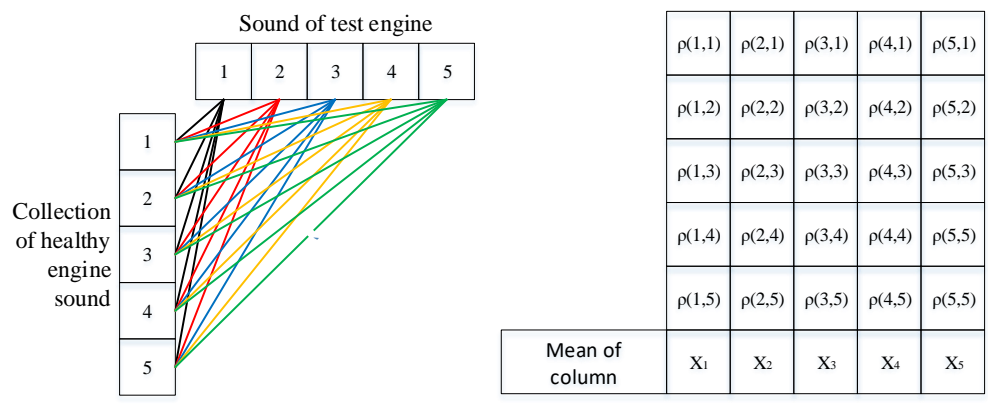

Figure 3. Matrix of correlation coefficients 


\section{RESULTS AND ANALYSIS}

\subsection{Frequency Pattern and Characteristic Value}

With the method mentioned above, we can get a frequency pattern of healthy engine, knocking engine, and hunting engine. Those 3 frequency pattern have defferences between them. For healthy engine the high power exist on $0 \mathrm{~Hz}-5.000 \mathrm{~Hz}$, and then after $5.000 \mathrm{~Hz}$ the power are relatively low and steady. Hunting engine the high power exist on $0 \mathrm{~Hz}-5.000 \mathrm{~Hz}$, then between $5.000 \mathrm{~Hz}$ and $15.000 \mathrm{~Hz}$ the power are low with some power increase between them, the over $15.000 \mathrm{~Hz}$ the power increase again. Knocking engine the high power exist on $0 \mathrm{~Hz}-15.000 \mathrm{~Hz}$ and relatively steady, then after $15.000 \mathrm{~Hz}$ the power drop and steady until $22.000 \mathrm{~Hz}$. Those 3 pattern have high power between $0 \mathrm{~Hz}-5.000 \mathrm{~Hz}$, it is due to the sound frequency of engine sound it self. It is said that the engine sound frequency that penetrate engine structural body are approximately $3.000 \mathrm{~Hz}$ [18]. Figure 4 and Figure 5 show those 3 frequency pattern.

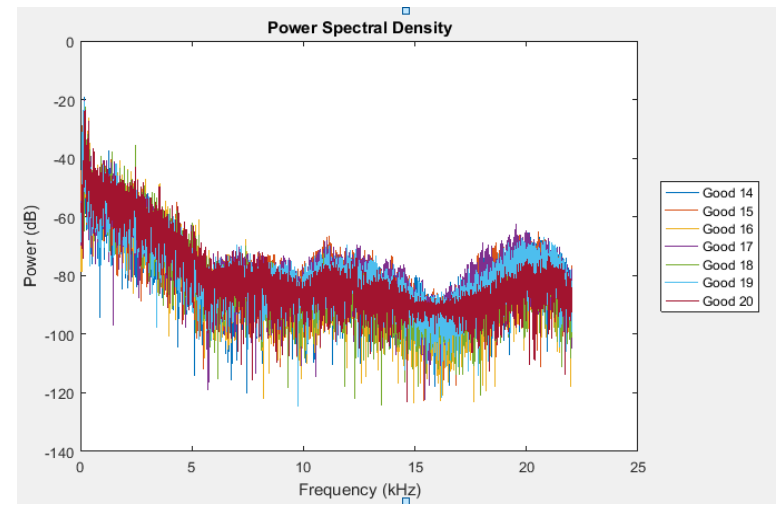

Figure 4. Frequency pattern of healthy engine

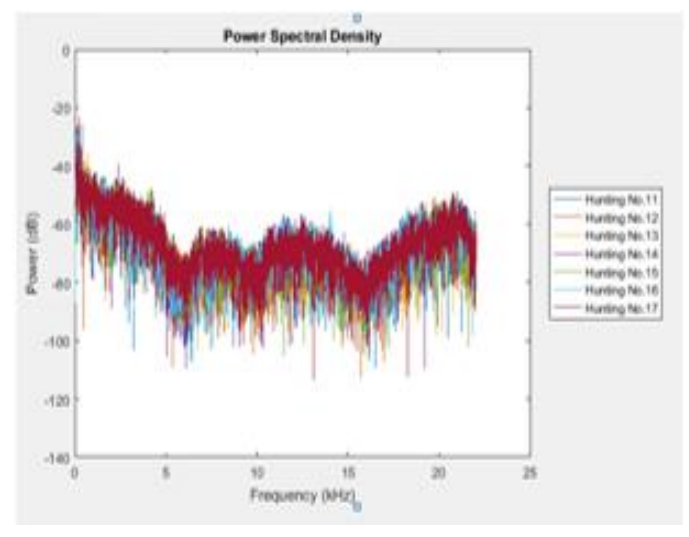

(a)

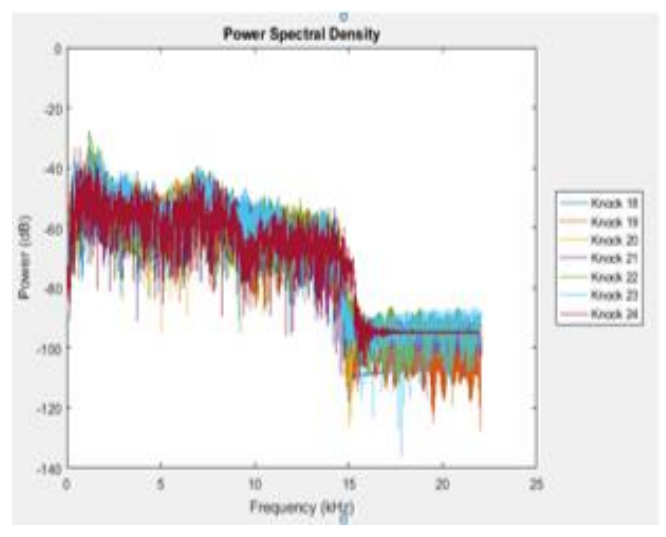

(b)

Figure 5. (a) Frequency pattern of hunting engine, (b) Frequency pattern of knocking engine

From frequency pattern on Figure 4, a value is generated using pattern comparison based on correlation coefficient method. The sample of engine sound are being tested a couple of time to get a characteristic value of healthy engine, knocking engine, and hunting engine. Those characteristic value are 0.75 for healthy engine, $0.6-0.7$ for hunting engine, and $0.4-0.65$ for knocking engine. Those value are used as follows:

a) If the comparison average value above 0.75 , then the engine in healthy condition. if the comparison average value below 0.75 , then the engine in damaged condition.

b) If the comparison average value between $0.6-0.7$, then the engine is in damaged condition and caused by hunting.

c) If the comparison average value between $0.4-0.65$, then the engine is in damaged condition and caused by knocking. 


\subsection{Research Result}

Test have been conducted several time using the characteristic value, as result from 85 acoustic sound signal most of the time, engine condition was successfully detected between healthy engine and damaged engine. Unfortunately, for detection of damage type the result are not satisfactory. The research result are from 36 healthy engine sound it was sucessfully detect 35 - 36 healthy engine sound, from 23 knocking engine sound it was successfully detect $8-12$ knocking engine sound, from 26 hunting engine sound it was successfully detect $13-17$ hunting engine sound. Table 3 gives the result of the research.

Table 3. Result of Engine Condition Detection

\begin{tabular}{cccccc}
\hline \multirow{2}{*}{ Number of Test } & Healthy & Broken & $\begin{array}{c}\text { Detected } \\
\text { Hunting }\end{array}$ & Knocking & Undefined \\
\hline 1 & 36 & 49 & 16 & 10 & 23 \\
2 & 36 & 49 & 16 & 9 & 24 \\
3 & 36 & 49 & 13 & 11 & 25 \\
4 & 36 & 49 & 17 & 10 & 22 \\
5 & 36 & 49 & 17 & 12 & 20 \\
6 & 36 & 49 & 14 & 11 & 24 \\
7 & 35 & 50 & 15 & 11 & 24 \\
8 & 36 & 49 & 15 & 10 & 24 \\
9 & 35 & 50 & 14 & 8 & 28 \\
10 & 36 & 49 & 13 & 10 & 26 \\
\hline
\end{tabular}

\section{CONCLUSION}

The conclusion from this research with proposed method are that the generated frequency patterns between three engine conditions showing distinctive differences. Those frequency patterns are processed using mathematical method to generate characteristic values which could reveal the engine condition between healthy engines or damaged engine. Unfortunately, those characteristic values still cannot give satisfactory result to detect the type of damage experienced by an engine.

With more sound samples from different engine manufacturers and models, this method can be used more universal to detect various engine sound. Any improvement can be made to make this research more useful. For example, by adding real time monitoring feature on smartphone platform that can be use to detect an engine condition on the spot.

\section{REFERENCES}

[1] Patricia Henriquez, Jesus B. Alonso, Miguel A. Ferrer, \& Carlos M. Travieso. (2014). Review of Automatic Fault Diagnosis Systems Using Audio and Vibration Signals. IEEE Transaction on Systems, Man, and Cybernetics: Systems, 44 (4), $642 \sim 652$.

[2] Sandeep Kumar Yadav, Kanishka Tyagi, Brijeshkumar Shah, \& Prem Kumar Kalra. (2011). Audio SignatureBased Condition Monitoring of Internal Combustion Engine Using FFT and Correlation Approach. IEEE Transaction on Instrumentation and Measurement, 60 (4), $1217 \sim 1226$.

[3] R. M. Vilela, J. C. Metrolho, \& J. C. Cardoso. (2004). Machine and Industrial Monitorization System by Analysis of Acoustic Signatures. Proceedings of the 12th IEEE Mediterranean Electrotechnical Conference, (pp. 277 279). Portugal: Escola Superior de Tecnologia, Instituto Politecnico de Castelo Branco.

[4] Shuaishi Liu, Min Yang, Keping Liu, \& Chen Chen. (2010). Research on Feature Extraction of Engine Abnormal Sound Signal Based on Linear Prediction Analysis. IEEE International Conference on Computer, Mechatronics, Control and Electronic Engineering, (pp. 76 79). Changchun, China: Changchun University of Technology.

[5] Hongjiang He, Chunxia Wang, \& Yazhou Wu. (2010). Application of Wavelet Transform in Sound Source Detection and Fault Diagnostic. IEEE International Conference on Computer, Mechatronics, Control and Electronic Engineering, (pp. 298 301). Changchun, China: Hebei University of Engineering.

[6] Mekala N, Muniraj C, Ramesh Balaji S.M. (2015). Vibration and Noise Analysis of 4Ф Switched Reluctance Motor Drive. TELKOMNIKA Indonesian Journal of Electrical Engineering, 14 (3), 410 419.

[7] Zhongjie Wang, Jingnan Zhang, Yongchun Liang. (2013). Motor Noise and Vibration Test Research. TELKOMNIKA Indonesian Journal of Electrical Engineering, 11 (1), 87 94.

[8] Selva Balan, Arti Khaparde, Vanita Tank, Tejashri Rade, \& Kirti Takalkar. (2014). Under Water Noise Reduction Using Wavelet and Savitzky-Golay. Second International Conference on Computational Science and Engineering, (pp. 243 250). Maharashtra, India: Maharashtra Institute of Technology.

[9] Li Jun, Dong Xingwen, Song Yaji, \& Song Nuan. (2013). Study on Engine Fault Diagnosis Based on the Abnormal Sound Analysis in Transformation Domain. Fourth IEEE International Conference on Digital Manufacturing \& Automation, (pp. 1272 1275). Qingdao, China: Air Force Aviation University. 
[10] Hocine Bendjama, Salah Bouhouche, \& Mohamed Seghir Boucherit. (2012). Application of Wavelet Transform for Fault Diagnosis in Rotating Mechinery. International Journal of Machine Learning and Computing, 2 (1), $82 \sim 87$.

[11] Cao Shuhua, Xu Junjun, \& Ning Dayong. (2015). Air Valve Clearance Fault Diagnosis of Diesel Engine Based on Acoustic Signal Data Processing. International Conference on Mechatronics, Electronics, Industrial and Control Engineering (MEIC), (pp. 1256 1259). Dalian, China: Dalian Maritime University.

[12] Qiang Wang, Xuemin Tian. (2013). Soft Sensing Based on Hilbert-Huang Transform and Wavelet Support Vector Machine. TELKOMNIKA Indonesian Journal of Electrical Engineering, 11 (7), 3704 3710.

[13] H Li, P Zhou, \& X Ma. (2005). Patern Recognition on Diesel Engine Working Condition by Using a Novel Methodology - Hilbert Spectrum Entropy. Journal of Marine Engineering \& Techology, 4 (1), 43-48.

[14] Michael Feldman. (2011). Hilbert Transform in Vibration Analysis. Mechanical Systems and Signal Processing, 25 (3), 735-802.

[15] Michael Feldman. (2006). Time-Varying Vibration Decomposition and Analysis Based on the Hilbert Transform. Journal of Sound and Vibration, 295 (3), 518-530.

[16] Steven W. Smith. (1997). The Scientist and Engineer's Guide to Digital Signal Processing. California: California Technical Publishing.

[17] Dr. M. J. de Smith. (2015). Statistical Analysis Handbook. United Kingdom: The Winchelsea Press

[18] Klaus Mollenhauer, Helmut Tschoeke. (2010). Handbook of Diesel Engine. London: Springer. 